\title{
A CLASS OF EXTREME LATTICE-COVERINGS OF $n$-SPACE BY SPHERES
}

(Received, 16 December 1970)

E. S. BARNES and D. W. TRENERRY

Communicated by G. E. Wall

All extreme lattice-coverings of $n$-space by spheres are known for $n \leqq 4$; see for example [3]. Only one class of extreme covering is known for large $n$, namely that corresponding to the quadratic form

$$
\phi_{0}(x)=n \sum_{i=1}^{n} x_{i}^{2}-2 \sum_{i<j} x_{i} x_{j}
$$

this was first shown to be extreme for all $n \geqq 2$ by Bleicher [2].

The object of this paper is to exhibit a new extreme lattice-covering for all odd $n \geqq 5$. The density of the covering is slightly larger than that corresponding to $\phi_{0}$, so that unfortunately no further information is provided on the density of the most economical covering.

We use the notation and some results of Voronoi $[5,6]$ and Barnes and Dickson [1]; a brief description of these follows.

Let $f(\boldsymbol{x})=f\left(x_{1}, x_{2}, \cdots, x_{n}\right)=\boldsymbol{x}^{\prime} A \boldsymbol{x}$ be positive definite, with determinant $d=d(f)$. Define the inhomogeneous minimum $m(f)$ of $f$ by

$$
m(f)=\max _{\alpha} \min _{\boldsymbol{x}} f(x+\alpha) \quad(\alpha \text { real, } x \in \Gamma)
$$

(where $\Gamma$ denotes the integral lattice) and define

$$
\mu(f)=m(f) / d^{1 / n} .
$$

If $A=P^{\prime} P$ and $\Lambda=\{P \boldsymbol{x} \mid \boldsymbol{x} \in \Gamma\}$, then spheres of radius $(m(f))^{\frac{1}{2}}$ centred the points of $\Lambda$ cover space minimally, and the density of the covering is

$$
\theta(\Lambda)=J_{n}(\mu(f))^{n / 2},
$$

where $J_{n}$ is the volume of the unit sphere.

We say that $f$ (and the corresponding covering) are extreme if $\mu(g) \geqq \mu(f)$ for all forms $g$ sufficiently close to $f$; if $f$ is extreme, so of course is any from equivalent to a multiple of $f$, and equivalent forms correspond to the same lattice covering. 
The Voronoi polytope $\Pi$ corresponding to $f$ is the set of points $\boldsymbol{x}$ satisfying

$$
f(\boldsymbol{x}) \leqq f(\boldsymbol{x}-\boldsymbol{l}) \quad \text { for all } \boldsymbol{l} \in \Gamma .
$$

A finite set $\pm l_{1}, \cdots, \pm \boldsymbol{l}_{\sigma}$ of integral points suffices to define $\Pi$, which has therefore $\sigma$ pairs of opposite parallel faces, with equations

$$
f(x)=f\left(x \pm l_{i}\right) \quad(i=1, \cdots, \sigma) .
$$

A given $l \neq 0$ belongs to this set iff

$$
f(l)=\min f(x)
$$

taken over all integral $\boldsymbol{x} \equiv \boldsymbol{l}(\bmod 2 \Gamma)$ and this minimum is attained only at $x= \pm \boldsymbol{l}$; we call these points the 'modulo $2 \Gamma$ minima' of $f$. Always $\sigma \leqq 2^{n}-1$, and in general $\sigma=2^{n}-1$, with one pair of faces for each class of $\Gamma / 2 \Gamma$ other than 0; such a form we call an interior form From the convexity of $\Pi$, it follows easily that

$$
m(f)=\max _{\boldsymbol{x} \in \Pi} f(\boldsymbol{x})=\max _{\boldsymbol{v}} f(\boldsymbol{v})
$$

where the maximum is taken over all vertices $v$ of $\Pi$. For an interior form, $\Pi$ is primitive (i.e. each vertex lies on just $n$ faces) and has $(n+1)$ ! vertices. A vertex $v$ for which the maximum in (1.5) is attained is said to be maximal.

Two vertices of $\Pi$ are congruent if they are congruent modulo $\Gamma$. Each vertex $v$ has $n+1$ congruent vertices; specifically, if $v$ lies on the $n$ planes

$$
f(x)=f\left(x-l_{i}\right) \quad(i=1, \cdots, n)
$$

we say that $v$ is determined by the simplex $\left[\boldsymbol{l}_{0}, \boldsymbol{l}_{1}, \cdots, \boldsymbol{l}_{n}\right]$ with vertices $\boldsymbol{l}_{0}$ $=\mathbf{0}, \boldsymbol{l}_{1}, \cdots, \boldsymbol{l}_{n}$. Then, for each $j=0,1, \cdots, n$, there is a congruent vertex $v_{j}=v-l_{j}$ of $\Pi$ determined by the simplex

$$
\left[l_{0}-l_{j}, l_{1}-l_{j}, \cdots, l_{\imath}-l_{j}\right] .
$$

Moreover, $f$ takes the same value at congruent vertices, so that all are maximal if any one is.

If $\boldsymbol{v}$ is determined by the simplex $\left[\boldsymbol{l}_{0}, \boldsymbol{l}_{1}, \cdots, \boldsymbol{l}_{n}\right]$, let $\left(c_{0}, c_{1}, \cdots, c_{n}\right)$ be its barycentric coordinates with respect to this simplex, so that

$$
v=\sum_{i=0}^{n} c_{i} l_{i}, \quad \sum_{i=0}^{n} c_{i}=1 .
$$

We then have (Barnes and Dickson [1]):

THEOREM 1. If $f(\boldsymbol{x})=\boldsymbol{x}^{\prime} A \boldsymbol{x}$ is an interior form and $\boldsymbol{F}(\boldsymbol{x})=\boldsymbol{x}^{\prime} A^{-1} \boldsymbol{x}$ is its inverse, then $f$ is extreme if and only if $F$ is expressible in the form 


$$
F(x)=\sum_{v} \lambda_{v}\left[\sum_{i=1}^{n} c_{i}\left(l_{i}^{\prime} x\right)^{2}-\left(v^{\prime} x\right)^{2}\right]
$$

where the outer sum is over all maximal vertices of $\Pi$,

$$
\lambda_{v} \geqq 0 \quad \text { for all } v,
$$

and, for each $v$, the $c_{i}$ are defined by (1.7).

We note also the corollary, that in (1.8) it suffices to include in the sum only one vertex $v$ from any set of $n+1$ congruent maximal vertices.

In $\S 2$ we describe the from $\phi_{2}(x)$ and prove that it is extreme for all odd $n \geqq 5$. Finally in $\$ 3$ we examine the density of the corresponding lattice-covering of space, and outline the genesis of the form and its relation to Voronoi's dissection of the space of positive definite quadratic forms into polyhedral cones.

\section{2}

THEOREM 2. For odd $n \geqq 5$, the form

$$
\phi_{2}(x)=\sum_{i=2}^{n} x_{i}^{2}+\sum_{2 \leqq i<j \leqq n}\left(x_{i}-x_{j}\right)^{2}+b \gamma(x)
$$

is extreme, where

$$
4 \gamma(x)=n\left(x_{1}^{2}+x_{2}^{2}\right)+4 \sum_{i=3}^{n} x_{i}^{2}+2(n-2) x_{1} x_{2}-4\left(x_{1}+x_{2}\right) \sum_{j=3}^{n} x_{j},
$$

and $b=b_{n}$ is the positive root of

$$
3(n-3) x^{2}+\left(n^{2}-8 n-6\right) x-4 n(n+1)=0 .
$$

It is convenient to transform the coordinates by

$$
\boldsymbol{x}=T \boldsymbol{y}: \quad \begin{aligned}
& x_{1}=y_{1}+y_{2} \\
& x_{2}=y_{1}-y_{2} \\
& x_{i}=y_{1}-y_{i} \quad(3 \leqq i \leqq n)
\end{aligned}
$$

and consider the form

$$
g(y)=\phi_{2}(T y)=\sum_{1 \leqq i<j \leqq n}\left(y_{i}-y_{j}\right)^{2}+b \sum_{i=1}^{n} y_{i}^{2},
$$

with

$$
d(g)=d\left(\phi_{2}\right)(\operatorname{det} T)^{2}=b(n+b)^{n-1} .
$$

If $\Lambda=T^{-1} \Gamma=\left\{T^{-1} x \mid x \in \Gamma\right\}$, then it is easily seen that $y \in \Lambda$ if and only if 


$$
2 y \in \Gamma \quad \text { and } \quad 2 y_{1} \equiv 2 y_{2} \equiv \cdots \equiv 2 y_{n}(\bmod 2) .
$$

Thus integral $\boldsymbol{x}$ correspond to points $\boldsymbol{y}$ satisfying (2.5).

The Voronoi polytope $\Pi_{\phi_{2}}$ under this transformation becomes $T^{-1} \Pi_{\phi_{2}}=\Pi$, which is not to be confused with the polytope $\Pi$ of the form $g$. The $\bmod 2 \Gamma$ minima of $\phi_{2}$ become the $\bmod 2 \Lambda$ of $g$, that is, a given $\boldsymbol{m} \neq \mathbf{0}$ belongs to the set $\left\{ \pm \boldsymbol{m}_{1}, \cdots, \pm \boldsymbol{m}_{\boldsymbol{\sigma}}\right\}$ of $\bmod 2 \Lambda$ minima of $g$ if and only if $g(\boldsymbol{m})=\min g(\boldsymbol{y})$ taken over all $\boldsymbol{y} \equiv \boldsymbol{m}(\bmod 2 \Lambda)$ and this minimum is attained only at $\boldsymbol{y}= \pm \boldsymbol{m}$. Thus $\boldsymbol{m}=T^{-1} \boldsymbol{l}$, where $\boldsymbol{l}$ is a $\bmod 2 \Gamma \operatorname{minimum}$ of $\phi_{2}$.

LEMMA 1. Let $\mathrm{e}_{1}$ denote the $i$ th unit vector and set $m=\frac{1}{2}(n-1)$. The set of mod $2 \Lambda$ minima of $g$ consists of the points

$$
\begin{gathered}
\frac{1}{2}\left( \pm e_{1} \pm e_{2} \pm \cdots \pm e_{n}\right) \\
\pm\left(e_{i_{1}}+e_{i_{2}}+\cdots+e_{i_{r}}\right) \quad\left(1 \leqq i_{1}<i_{2} \cdots<i_{r} \leqq n, 1 \leqq r \leqq m\right) .
\end{gathered}
$$

Proof. For points of the form (2.6) it is sufficient, after applying a suitable permutation of coordinates, to consider a point of the form

$$
m=\frac{1}{2}\left(e_{1}+\cdots+e_{r}-e_{r+1}-\cdots-e_{n}\right)=\frac{1}{2}(1, \cdots, 1,-1, \cdots,-1) .
$$

From (2.5), any point congruent to $m \bmod 2 \Lambda$ is of the form

$$
\boldsymbol{y}=\frac{1}{2}\left(1+4 a_{1}, \cdots, 1+4 a_{r},-1+4 a_{r+1}, \cdots,-1+4 a_{n}\right)
$$

or

$$
\boldsymbol{y}=\frac{1}{2}\left(-1+4 a_{1}, \cdots,-1+4 a_{r}, 1+4 a_{r+1}, \cdots, 1+4 a_{n}\right),
$$

with integral $a_{1}, \cdots, a_{n}$. Over points of the form (2.8), $\Sigma y_{i}^{2}$ attains its minimum only when $a_{1}=\cdots=a_{n}=0$ and each $\left(y_{i}-y_{j}\right)^{2}$ also takes its minimun value there, so that $g(y)$ attains its minimum only at $\boldsymbol{m}$. Similarly, over points of the form (2.9), $g(y)$ attains its minimum only at $-\boldsymbol{m}$. Thus all points (2.6) are $\bmod 2 \Lambda$ minima of $g$.

For points (2.7) it suffices to consider

$$
\boldsymbol{m}=e_{1}+\cdots+e_{r}=(1, \cdots, 1,0, \cdots, 0),
$$

where $1 \leqq r \leqq m$. From (2.5), any point congruent to $m \bmod 2 \Lambda$ is of the form

$$
\begin{aligned}
& \boldsymbol{y}=\left(1+2 a_{1}, \cdots, 1+2 a_{r}, 2 a_{r+1}, \cdots, 2 a_{n}\right) \\
& \boldsymbol{y}=\left(2 a_{1}, \cdots, 2 a_{r}, 2 a_{r+1}-1, \cdots, 2 a_{n}-1\right),
\end{aligned}
$$

with integral $a_{1}, \cdots, a_{n}$. Points of the form (2.10) have $y_{i}-y_{j}$ even if $i \leqq r$ and $j \leqq r$ or $i>r$ and $j>r$, and odd if $i \leqq r$ and $j>r$; it is therefore easily seen that $\sum\left(y_{i}-y_{j}\right)^{2}$ attains its minimum $r(n-r)$ only when $a_{1}=\cdots=a_{n}=0$ or $a_{1}=\cdots=a_{r}=-1$ and $a_{r+1}=\cdots=a_{n}=0 . \sum y_{i}^{2}$ also attains its minimum $r$ at these points and so $g(y)$ attains its minimum $r(n-r)+r b$ over points (2.10) only 
at $\pm \boldsymbol{m}$. Similarly $g(y)$ attains its minimum $r(n-r)+(n-r) b$ over points $(2.11)$ only at $\pm(0, \cdots 0,1, \cdots, 1)$. Since $1 \leqq r \leqq \frac{1}{2}(n-1), r(n-r)+(n-r) b>r(n-r)$ $+r b$ and so the minimum of $g(y)$ over points congruent to $m \bmod 2 \Lambda$ is attained only at $\pm \boldsymbol{m}$; hence points $(2.7)$ are $\bmod 2 \Lambda$ minima of $g$.

There are $2^{n-1}$ pairs $\pm m$ of the form (2.6) and $2^{n-1}-1$ pairs of the form (2.7), each from a different class of $\Lambda / 2 \Lambda$. It follows that (2.6) and (2.7) give all the $\bmod 2 \Lambda$ minima of $g$.

Lemma 2. The set $S$

$$
\begin{aligned}
& \boldsymbol{e}_{1}+\cdots+\boldsymbol{e}_{r} \quad(1 \leqq r \leqq m) \\
& \frac{1}{2}\left(\boldsymbol{e}_{1}+\cdots+\boldsymbol{e}_{m}+\boldsymbol{e}_{m+1}+\cdots+\boldsymbol{e}_{m+s}-\boldsymbol{e}_{m+s+1}-\cdots-\boldsymbol{e}_{n}\right) \quad(0 \leqq s \leqq m),
\end{aligned}
$$

of mod $2 \Lambda$ minima of $g$ determines a vertex

$$
\begin{gathered}
v=\frac{1}{4(n+b)}(2 b+4 m+2 m-1,2 b+4(m-1)+2 m-1, \cdots, \\
2 b+4+2 m-1,2 m-1,2 m-1-4, \cdots, \\
2 m-1-4(m-1), 2 m-1-4 m-b)
\end{gathered}
$$

of $\Pi$, and every vertex of $\Pi$ is equivalent to $v$ or a vertex congruent to $v$.

Proof. By $I$ we denote the unit matrix and by $K$ the matrix with components $k_{i i}=0(1 \leqq i \leqq n)$ and $k_{i j}=1(1 \leqq i, j \leqq n, i \neq j)$, so that

$$
a I+b K=\left[\begin{array}{cccccc}
a & b & \cdot & \cdot & \cdot & b \\
b & a & & & \cdot \\
\cdot & & \cdot & & \cdot \\
\cdot & & & \cdot & \cdot \\
\cdot & & & & \cdot & b \\
b & \cdot & \cdot & \cdot & b & a
\end{array}\right] .
$$

With this notation, the matrix $A$ of $g$ is $(n-1+b) I-K$ and so

$$
A^{-1}=\frac{1}{b(n+b)}((1+b) I+K) \text {. }
$$

$\Pi$ is determined by the inequalities

$$
\begin{array}{lll} 
& g(y) \leqq g(y-m) & (m \in S), \\
\text { that is } & 2 \boldsymbol{m}^{\prime} A y \leqq g(\boldsymbol{m}) & (\boldsymbol{m} \in S) .
\end{array}
$$

Setting, for convenience,

$$
\boldsymbol{z}=\boldsymbol{A} \boldsymbol{y}
$$

then, since $\boldsymbol{m} \in S$ implies $-\boldsymbol{m} \in S$, the inequalities are

$$
2\left|\boldsymbol{m}^{\prime} z\right| \leqq g(\boldsymbol{m}) \quad(\boldsymbol{m} \in S),
$$


that is

$$
2\left|z_{i_{1}}+\cdots+z_{i_{r}}\right| \leqq r(n-r)+r b \quad\left(1 \leqq i_{1}<\cdots<i_{r} \leqq n, 1 \leqq r \leqq m\right)
$$

$$
\left|z_{1} \pm \cdots \pm z_{n}\right| \leqq k(n-k)+\frac{n}{b} b \quad(k=\text { number of minus signs }) .
$$

The $n$ faces of $\Pi$ determined by (2.12) are

$$
2\left(z_{1}+\cdots+z_{r}\right)=r(n-r)+r b \quad(1 \leqq r \leqq m)
$$

$$
\begin{aligned}
\left(z_{1}+\cdots+z_{m}\right. & \left.+\cdots+z_{m+s}-z_{m+s+1}-\cdots-z_{n}\right) \\
& =(m+1-s)(m+s)+\frac{n}{4} b \quad(0 \leqq s \leqq m) .
\end{aligned}
$$

These intersect at the point

$$
z=\frac{1}{2}\left(2 m+b, 2 m-2+b, \cdots, 2+b, 0,-2, \cdots,-(2 m-2),-2 m-\frac{1}{2} b\right),
$$

which is easily seen to satisfy the inequalities (2.16) with equality only in (2.17). Hence $z$ is a vertex of the region defined by (2.16), and $v=A^{-1} z$ is a vertex of $\Pi$.

Since, by (2.15) and (2.14),

$$
\begin{aligned}
v & =\frac{1}{b(n+b)}((1+b) I+K) z \quad \text { and } \quad \sum_{i=1}^{n} z_{i}=\frac{1}{4}(2 m-1) b \\
v_{i} & =\frac{1}{b(n+b)}\left(b z_{i}+z_{1}+\cdots+z_{n}\right) \\
& =\frac{1}{b(n+b)}\left(b z_{i}+\frac{1}{4}(2 m-1) b\right) \\
& =\frac{1}{n+b}\left(z_{i}+\frac{1}{4}(2 m-1)\right)
\end{aligned}
$$

and the expression (2.13) results.

Permuting suffixes in (2.12) gives $n$ ! distinct vertices of $\Pi$ equivalent to $v$. The $n$ vertices congruent to $v$ (other than $v$ ) are the points $v-m$ with $\boldsymbol{m} \in S$, which are easily seen to be distinct from these. Hence we have $(n+1)$ ! distinct vertices of $\Pi$ and therefore all vertices of $\Pi$.

ProOF of TheOREM. We shall prove the equivalent result, that $g(v)$ is extreme over $\Lambda$, by using the Theorem 1 .

Writing, as in (1.7), $v=\sum_{i=1}^{n} c_{i} \boldsymbol{m}_{i}$, we deduce from (2.12) that

$$
\begin{aligned}
c_{i} & =v_{i}-v_{i+1} \\
c_{m} & =v_{m}+v_{n}
\end{aligned}
$$




$$
\begin{aligned}
c_{m+1} & =-v_{m+1}-v_{n} \\
c_{m+j+1} & =v_{m+j}-v_{m+j+1} \quad(1 \leqq j \leqq m)
\end{aligned}
$$

whence

$$
c=\frac{1}{4(n+b)}(4, \cdots, 4,2+b, 4+b, 4, \cdots, 4,2+b) .
$$

Also write

$$
\begin{gathered}
\psi(\boldsymbol{y})=\sum_{i=1}^{n} c_{i}\left(\boldsymbol{m}_{i}^{\prime} \boldsymbol{y}\right)^{2}-\left(\boldsymbol{v}^{\prime} \boldsymbol{y}\right)^{2} \\
=\alpha_{11} y_{1}^{2}+\cdots+\alpha_{n n} y_{n}^{2}+2 \alpha_{12} y_{1} y_{2}+\cdots+2 \alpha_{n-1, n} y_{n-1} y_{n} .
\end{gathered}
$$

Since all vertices are equivalent or congruent, all vertices are maximal and the sum (1.8) is over all vertices. By the Corollary to Theorem 1 it suffices to sum over all vertices equivalent to $v$, i.e. to sum over all permutations of coordinates of $\boldsymbol{v}$.

Summing (2.19) over all permutations of coordinates and counting the number of times terms appear, we obtain

$$
\begin{aligned}
& \sum_{\sigma \in S_{n}} \sigma(\psi(y))=(n-1) !\left(\sum_{i=1}^{n} \alpha_{i i}\right)\left(y_{1}^{2}+\cdots+y_{n}^{2}\right) \\
& +2(n-2) !\left(\sum_{i<j} \alpha_{i j}\right)\left(2 y_{1} y_{2}+\cdots+2 y_{n-1} y_{n}\right) \\
& =2(n-2) !\left\{\frac{1}{2}(n-1)\left(\sum_{i} \alpha_{i i}\right)\left(y_{1}^{2}+\cdots+y_{n}^{2}\right)\right. \\
& \left.+\left(\sum_{i<j} \alpha_{i j}\right)\left(2 y_{1} y_{2}+\cdots+2 y_{n-1} y_{n}\right)\right\} .
\end{aligned}
$$

We note that

so that

$$
\psi(1, \cdots, 1)=\sum_{i} \alpha_{i i}+2 \sum_{i<j} \alpha_{i j}
$$

$$
2 \sum_{i<j} \alpha_{i j}=\psi(1, \cdots, 1)-\sum_{i} \alpha_{i i} .
$$

Hence, from (2.20), $\sum_{\sigma \in S_{n}} \sigma(\psi(y))$ has matrix

$$
B=2(n-2) !\left\{\frac{1}{2}(n-1)\left(\sum_{i} \alpha_{i i}\right) I+\frac{1}{2}\left(\psi(1, \cdots, 1)-\sum_{i} \alpha_{i i}\right) K\right\} \text {. }
$$

Substituting (2.13) and (2.18) into (2.19) gives

$$
\begin{array}{r}
48(n+b)^{2} \psi(1, \cdots, 1)=b^{2}\left(12 m^{2}+3\right)+b\left(32 m^{3}+24 m^{2}+16 m+6\right) \\
+16 m^{4}+32 m^{3}+32 m^{2}+16 m+3,
\end{array}
$$




$$
\begin{aligned}
C=48(n+b)^{2} \sum_{i=1}^{n} \alpha_{i i}= & b^{2}(12 m+3)+b\left(48 m^{2}+36 m+6\right) \\
& +40 m^{3}+60 m^{2}+26 m+3
\end{aligned}
$$

and, by (2.21),

$$
\begin{aligned}
2 D & =48(n+b)^{2} 2\left(\alpha_{12}+\cdots+\alpha_{n-1, n}\right) \\
& =b^{2}\left(12 m^{2}-12 m\right)+b\left(32 m^{3}-24 m^{2}-20 m\right)+16 m^{4}-8 m^{3}-28 m^{2}-10 m,
\end{aligned}
$$

so that $B$ is a multiple of $m C I+D K$.

If $m C=(1+b) D$ then $B$ is a multiple of $(1+b) I+K$ and so by (2.14) is a multiple of $A^{-1}$. Thus positive constants

$$
\lambda_{v}=\frac{24(n+b)}{(n-2) ! D}
$$

can be chosen so that (1.8), with the sum taken over one vertex from each congruence class, is satisfied, and the theorem is proved.

The relation $m C=(1+b) D$ reduces to

$$
\begin{array}{r}
b^{3}(6 m-6)+b^{2}\left(16 m^{2}-18 m-19\right)+b\left(8 m^{3}-36 m^{2}-62 m-21\right) \\
-\left(32 m^{3}+64 m^{2}+40 m+8\right)=0
\end{array}
$$

that is,

(2.22) $(b+2 m+1)\left(b^{2}(6 m-6)+b\left(4 m^{2}-12 m-13\right)-\left(16 m^{2}+24 m+8\right)\right)=0$.

$b$ is the positive root of (2.2), which can be written in terms of $m=\frac{1}{2}(n-1)$ as

$$
x^{2}(6 m-6)+x\left(4 m^{2}-12 m-13\right)-\left(16 m^{2}+24 m+8\right)=0
$$

and so the relation $(2.22)$ is satisfied.

\section{3}

Since all vertices of $\Pi$ are maximal, $m\left(\varphi_{2}\right)=g(v)$, where $v$ is given by (2.13); a staightforward computation yields

$$
\text { (3.1) } \begin{aligned}
m\left(\varphi_{2}\right)=\frac{1}{48(n+b)}\left\{\left(32 m^{3}+48 m^{2}+16 m\right)+\left(36 m^{2}\right.\right. & +36 m+3) b \\
& \left.+(12 m+3) b^{2}\right\} .
\end{aligned}
$$

From (2.4), $d\left(\varphi_{2}\right)=b(n+b)^{n-1}$ so that $\mu\left(\varphi_{2}\right) / d^{1 / n}\left(\varphi_{2}\right)$ may now be calculated. We append a short table, in which we list for comparison the value of $\mu$ for the 'principal form' $\varphi_{0}$ defined in (1.1), namely

$$
\mu\left(\varphi_{0}\right)=\frac{1}{12} n(n+2)(n+1)^{-1+1 / n} .
$$




$\begin{array}{rccc}n & b_{n} & \mu\left(\varphi_{2}\right) & \mu\left(\varphi_{0}\right) \\ 5 & 6.552 & 0.7093 & 0.6956 \\ 7 & 4.896 & 0.8981 & 0.8832 \\ 9 & 4.390 & 1.0797 & 1.0655 \\ 11 & 4.162 & 1.2578 & 1.2447\end{array}$

As $n \rightarrow \infty, b_{n} \rightarrow 4$ and $\mu\left(\varphi_{2}\right) \sim \frac{1}{12} n$.

The polyhedral cone (see [6], [1, p. 117]), which we denote by $\Delta_{2}$, of which $\varphi_{2}$ is (apart from multiples) the unique extreme form, has the property that every interior form $f$ has

(i) the set of $\bmod 2$ minima specified in Lemma 1 ;

(ii) a polytope $\Pi(f)$ all of whose vertices are determined by the same simplices as those of $\Pi\left(\varphi_{2}\right)$ (as specified by Lemma 2). It may be shown that, explicitly, $\Delta_{2}$ is the set of forms $f(x)$ expressible as

$$
\sum_{i<j} \lambda_{i j}\left(y_{i}-y_{j}\right)^{2}+\sum_{i} \mu_{i} \chi_{i}(y)+\sum_{i} v_{i} \omega_{i}(y)
$$

with

$$
\lambda_{i j} \geqq 0 \quad(1 \leqq i<j \leqq n), \quad \mu_{i} \geqq 0, \quad v_{i} \geqq 0 \quad(1 \leqq i \leqq n) ;
$$

where

$$
\chi_{i}(y)=\sum_{j=1}^{n} y_{j}^{2}-y_{i}^{2}, \quad \omega_{i}(y)=\sum_{j=1}^{n} y_{j}^{2}+y_{i}^{2}
$$

and the variables $\boldsymbol{x}, \boldsymbol{y}$ are related by the transformation $T$ of $\S 2$.

The group of automorphisms of $\Delta_{2}$ is the full symmetric group $\mathscr{S}_{n}$, induced by all permutations of the variables $y_{1}, \cdots, y_{n}$; and the forms of $\Delta_{2}$ which are invariant under this group are those of the shape

$$
\lambda \sum_{i<j}\left(y_{i}-y_{j}\right)^{2}+\alpha \sum_{i} y_{i}^{2}
$$

According to the theorem of Dickson [4], an interior form $f$ of $\Delta_{2}$ is extreme if and only if if it is of the shape (3.2) (with $\lambda>0, \alpha>0$ ), and maximizes $\mu(f)$ over the set of such forms. It was this result which led to the consideration of a form of the type (2.3) and the determination of the equation (2.2) satisfied by $b_{n}$.

The cone $\Delta_{2}$ is also of independent interest in providing the first example of a Voronoi cone with more than $\frac{1}{2} n(n+1)$ edges. 


\section{References}

[1] E. S. Barnes and T. J. Dickson, 'Extreme coverings of $n$-space by spheres', J. Aust. Math. Soc. 7 (1967), 115-127. Corrigendum, ibid 8 (1968), 638-640.

[2] M. N. Bleicher, 'Lattice coverings of $n$-space by spheres', Can. J. Math. 14 (1962), 632-650.

[3] T. J. Dickson, 'The extreme coverings of 4-space by spheres', J. Aust. Math. Soc. 7 (1967), 490-496.

[4] T. J. Dickson, 'A sufficient condition for an extreme covering of $n$-space by spheres', J. Aust. Math. Soc. 8 (1968), 56-62.

[5] G. Voronoi, 'Recherches sur les paralléloèdres primitifs' (Part 1), J. reine angew. Math. 134 (1908), 198-287.

[6] G. Voronoi, 'Recherches sur les paralléloèdres primitifs' (Part 2), J. reine angew. Math. 136 (1909), 67-181.

Mathematics Department

University of Adelaide

Australia 